\title{
Estómago de Conejo: Modelo Animal para Cirugía Experimental
}

\author{
Rabbit Stomach: Animal Model for Experimental Surgery
}

"María Teresa Halabi; "Francisca Bahamondes; "Gino Cattaneo; **Luis Adaro \& "Estefanía Flores

HALABI, M. T.; BAHAMONDES, F.; CATTANEO, G.; ADARO, L. \& FLORES, E. Estómago de conejo: modelo animal para cirugia experimental. Int. J. Morphol., 30(1):82-87, 2012.

RESUMEN: La cirugía veterinaria experimental en pequeños animales, particularmente en perro, tiene principalmente restricciones de tipo afectivas, por lo que es necesario contar con otro modelo animal, para poder realizar diferentes procedimientos destinados a su aplicación en dicha especie. Dicho modelo debe cumplir con características anatómicas homologables al perro y también con facilidades de uso y manejo. Al ser una especie tradicionalmente utilizada en experimentación, el conejo podría constituir un excelente modelo animal para este propósito. El objetivo de este estudio es describir la anatomo-topografía del estómago del conejo para luego compararla con el perro. Se utilizaron 14 conejos que fueron sometidos a distintas técnicas de conservación para su estudio anatómico posterior; los instrumentos y materiales para realizar dicho estudio morfológico y un registro bibliográfico de parámetros anatómicos del estómago del perro. Los resultados de este ensayo muestran que el conejo posee un estómago de morfología, ubicación y orientación similar a la del perro, con algunas variaciones en sus relaciones con otras estructuras anatómicas. Asimismo la irrigación de este órgano es muy similar a la del perro. De acuerdo a lo observado, en estómago, el conejo podría ser utilizado como modelo animal para someter a prueba distintos ensayos quirúrgicos experimentales.

PALABRAS CLAVE: Conejo; Morfología; Estómago; Modelo Animal.

\section{INTRODUCCIÓN}

En la cirugía experimental se someten a prueba distintos procedimientos, tanto innovadores como tradicionales, estos últimos principalmente para docencia. Los ensayos de este tipo en animales de compañía, como lo es el perro, tienen restricciones de tipo ético y emocional (experimentar en una especie con la que existen lazos afectivos) que complicarían su uso como modelo en investigación y entrenamiento quirúrgico. Por esto surge la necesidad de buscar especies alternativas que permitan las realización de procedimientos quirúrgicos experimentales para entrenamiento en docencia, con menos restricciones en su uso. Dicha especie debe cumplir con características anatómicas similares.

El conejo, siendo ampliamente usado en experimentación y docencia, podría cumplir con las características necesarias. Es un animal de abasto, de fácil manejo y cuidado, no requiere de gran espacio físico y su alimentación es simple. No obstante, no ha sido explorada la posibilidad de su uso como modelo experimental en procedimientos que requieren de similitud morfológica, para que sus resultados puedan ser extrapolables a otra especie.

Este estudio buscó establecer al conejo como un modelo animal para aplicación quirúrgica experimental y docente. Se comparó tanto la morfología como la irrigación arterial del estómago del conejo versus la del perro, en técnicas de gran importancia en la formación quirúrgica general, para los médicos veterinarios. Las cirugías digestivas representan un 7,16\% del total de cirugías que se realizan (Ramírez, 2002), siendo este sistema uno de los tres más intervenidos, junto al urogenital y esquelético.

\section{MATERIAL Y MÉTODO}

Se utilizaron 14 cadáveres de conejos mestizos, con un rango de peso entre los 2 y $3 \mathrm{~kg}$, provenientes de actividades docentes experimentales, de los cuales 10 cadáveres

\footnotetext{
"Departamento de Cs Clínicas, Facultad de Ciencias Veterinarias y Pecuarias, Universidad de Chile, Santiago, Chile. *Departamento de Cs Biológicas, Facultad de Ciencias Veterinarias y Pecuarias, Universidad de Chile, Santiago, Chile.
} 
se inyectaron con una solución de fijación y 4 se inyectaron con látex coloreado y se conservaron para su estudio posterior.

En los diez especímenes inyectados con solución fijadora se realizó una incisión mediana amplia, que se extendió desde los procesos xifoides hasta el margen craneal del pubis. Luego se desplazó la pared abdominal muscular ventral para dejar expuesto el estómago.

La metodología a seguir para el material biológico, conservado en solución fijadora inyectada, fue la observación directa, y se midieron los siguientes parámetros anatomo-quirúrgicos- de interés: Relaciones topográficas del estómago con elementos anatómicos adyacentes y presencia de estructuras anatómicas y su morfología.

Para obtener el material biológico inyectado con látex coloreado se realizó la técnica de Tompsett (1970), en cadáveres de animales previamente heparinizados (en una dosis 200 UI/ Kg) eutanasiados con sobredosis de Tiopental. Fueron procesados inmediatamente postmortem. Cada ejemplar se fijó con $500 \mathrm{ml}$ de formalina al $10 \%$ mediante inyec-

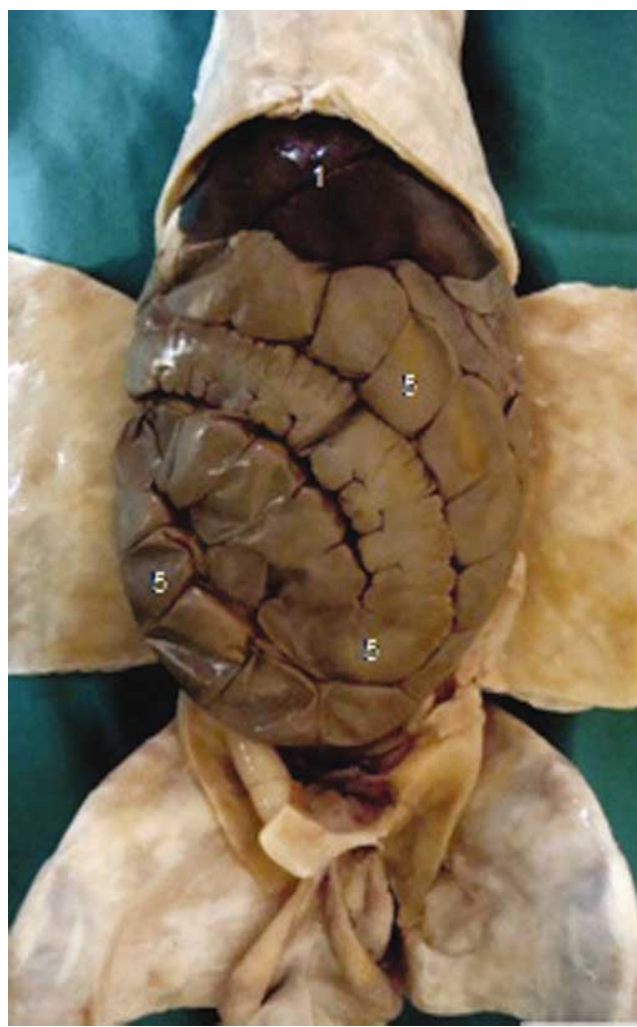

Fig. 1. Vista ventral de la cavidad abdominal del conejo. La pared abdominal ha sido desplazada y la primera estructura visible es el ciego. 1. Hígado. 2. Estómago. 3. Duodeno. 4. Yeyuno. 5. Ciego. 6. Intestino grueso. ciones intraperitoneales e intramusculares y se refrigeró durante 3 días previa disección de los vasos sanguíneos. Se disecaron los troncos arteriales principales asociados al estómago para su posterior identificación y descripción en ambos casos (material biológico inyectado en solución de fijación e inyectados en látex) se realizó:

- Registro de imágenes a color, siendo la observación directa registrada con fotografías.

- Comparación de estructuras con los parámetros anatómicos homólogos del perro, según referencias bibliográficas.

\section{RESULTADOS Y DISCUSIÓN}

Descripción y comparación anatómica del estómago de conejo y perro. Al comparar al conejo con el perro podemos observar que, al realizar una incisión mediana amplia con posterior desplazamiento de la pared muscular ventral del abdomen (Figs. 1 y 2), existen diferencias en relación a las primeras estructuras visibles.

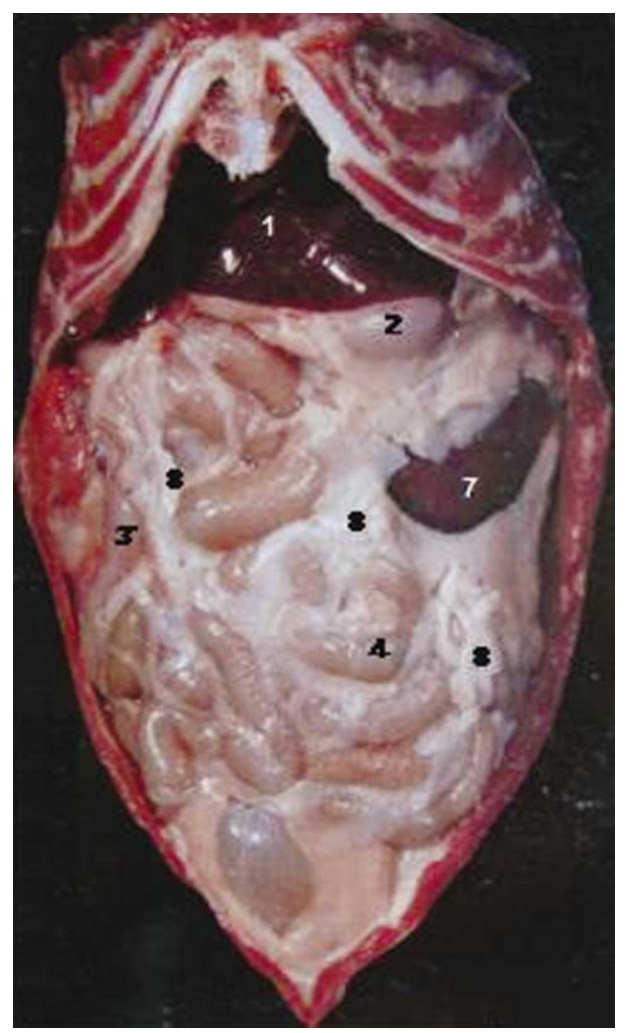

Fig. 2. Vista ventral de la cavidad abdominal del perro. La pared abdominal ha sido desplazada (Ruberte, 1995). 1. Hígado. 2. Estómago. 3. Duodeno. 4. Yeyuno. 5. Ciego. 6. Intestino grueso. 7. Bazo. 8. Omento mayor. 
La primera estructura visible en el conejo es el ciego, el que cubre al estómago, obligando a desplazar esta estructura para poder ubicar el estómago. En el perro es el omento mayor el que cubre la masa visceral abdominal, esta estructura es muy grande, y en animales bien nutridos contiene mucha grasa. Observado ventralmente cubre toda la masa intestinal, y se extiende desde la curvatura mayor hasta la entrada pelviana (Sisson \& Grossman, 1982), a diferencia del conejo en donde el omento mayor es muy corto y no alcanza a cubrir la masa intestinal (Fig. 3).

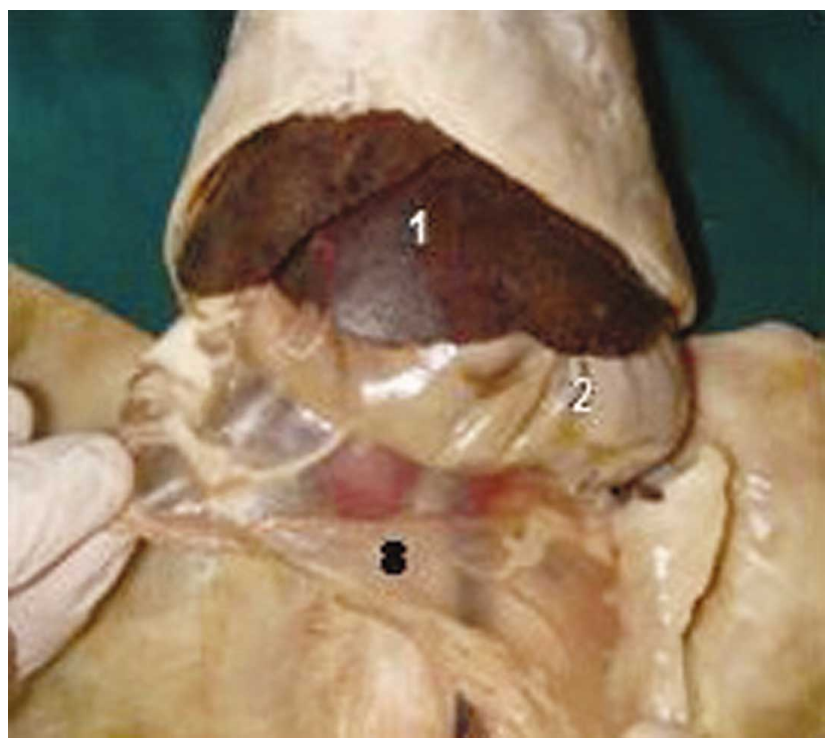

Fig. 3. Exposición Omento mayor del conejo. 1= Hígado, 2= Estómago, 3= Duodeno, 4= Yeyuno, 5= Ciego, 6= Intestino grueso, $7=$ Bazo, $8=$ Omento mayor.

El estómago del conejo se encuentra en la porción epigástrica del abdomen. Su curvatura mayor se dispone hacia la izquierda y ventral del plano mediano, y la curvatu- ra menor hacia la derecha y dorsal. El estómago se relaciona cranealmente con hígado, ventralmente y a su izquierda con ciego y colon descendente, a su izquierda con la porción craneal del riñón izquierdo, a la derecha con duodeno y caudalmente con intestino delgado, principalmente yeyuno. En la curvatura mayor del estómago se puede distinguir como emerge el omento mayor.

El estómago del conejo tiene una ubicación y orientación similar al del perro, variando en su relación con pared abdominal. En el perro se relaciona ventralmente con la pared abdominal (Sisson \& Grossman), en el conejo no existe esta relación debido a que el ciego se interpone entre estas dos estructuras.

El conejo está considerado como una especie monogástrica, al igual que los cerdos, las aves y los carnívoros. Anatómicamente se pueden distinguir dos sectores: primero la zona que incluye al cardias y el fondo, la cual actuaría como reservorio, segundo al antro, de mucosa glandular y paredes algo más gruesas (Gecele, 1986). El fondo tiene forma de cúpula y se encuentra orientado hacia la izquierda del plano medio y dorsal al cuerpo del estómago. Su pared muscular es delgada y flexible. El cuerpo se extiende desde el fondo hacia la derecha y termina en la región pilórica a nivel del hipocondrio derecho. El canal pilórico del conejo es de paredes musculares más gruesas que el resto del estómago y termina en el esfínter pilórico (Halabí, 2009).

Al ser ambas especies monogástricas la forma del estómago es similar (Figs. 4 y 5), presentando pequeñas diferencias destacables, como el tamaño y profundidad de las curvaturas mayor y menor, más pronunciadas en el perro que en el conejo.
Fig. 4. Cara visceral del estómago del conejo.

1. Esófago.

2. Cardias.

3. Fondo.

4. Cuerpo.

5. Canal pilórico.

6. Píloro.

7. Curvatura menor.

8. Curvatura mayor.

9. Inserción omento menor.

10. Inserción omento mayor.

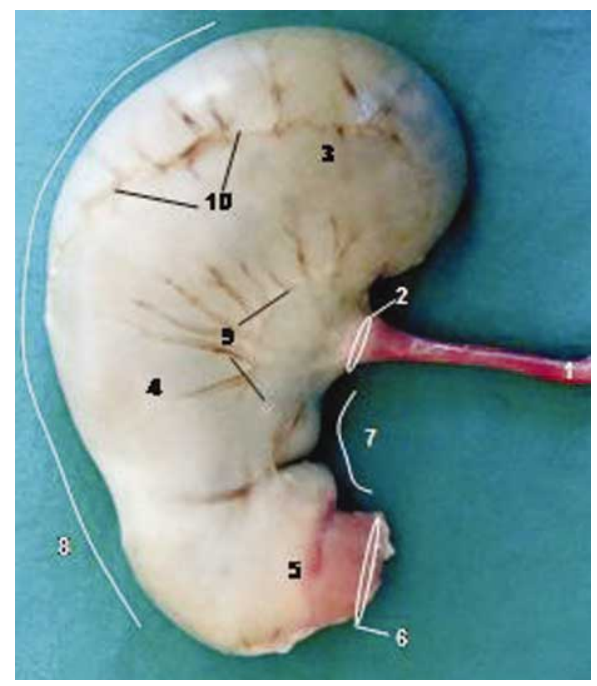

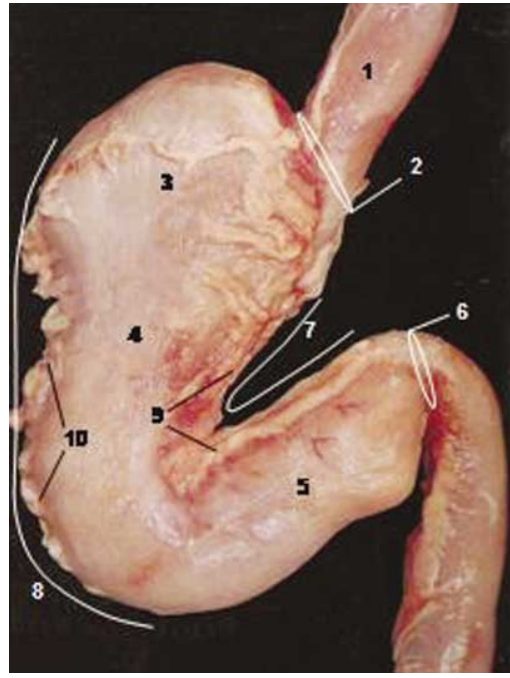

Fig. 5. Cara visceral del estómago del perro (Ruberte, 1995). 1. Esófago. 2. Cardias. 3. Fondo. 4. Cuerpo. 5. Canal pilórico. 6. Píloro.

7. Curvatura menor. 8. Curvatura mayor. 9. Inserción omento menor, 10. Inserción omento mayor. 
La zona cardial y fúndica del estómago del conejo es de paredes delgadas y poco musculosas, por lo tanto no hay contracciones que obliguen al contenido alimentario a llegar hasta la región pilórica (Amorim et al., 2001), es por esta razón que el tamaño del fondo en relación al tamaño del estómago, en el conejo es mayor que en el perro, ya que es más difícil que el estómago esté vacío, incluso con ayuno prolongado.

\section{Descripción y comparación de la irrigación gástrica en el conejo y el perro}

Arteria celiaca y sus ramas. La irrigación sanguínea del estómago de los carnívoros, como el perro, proviene de las tres ramas principales de la arteria celiaca: la arteria esplénica, la arteria gástrica izquierda y la arteria hepática, que dan origen a diversas ramas que irrigan el estómago y algunos órganos adyacentes. Esta irrigación es muy abundante, en particular a lo largo de las dos curvaturas (Dyce et al., 1999). Se observó que el conejo, al igual que en el perro la irrigación sanguínea arterial del estómago también proviene de la arteria celiaca y sus ramas (Figs. 6, 7 y 8).

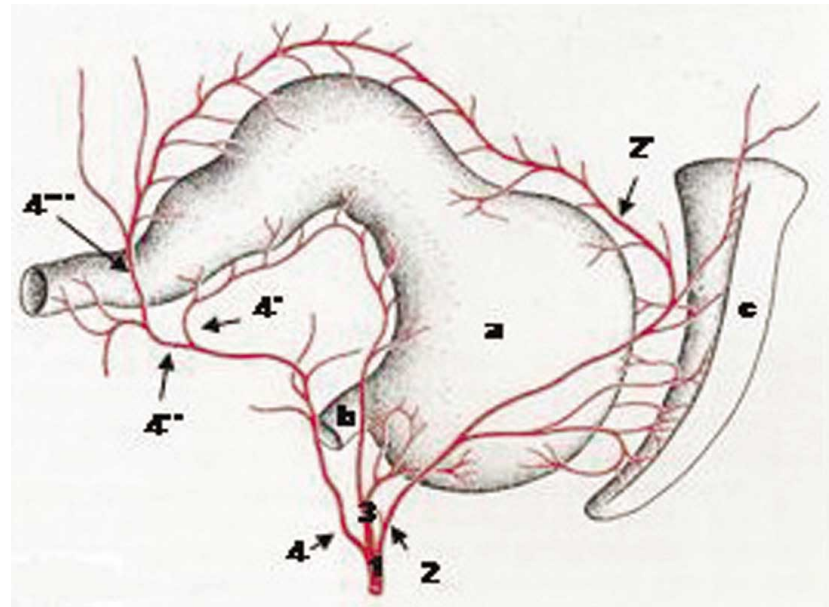

Fig. 6. Esquema de la irrigación arterial de la cara visceral del estómago del perro (Nickel et al., 1976).

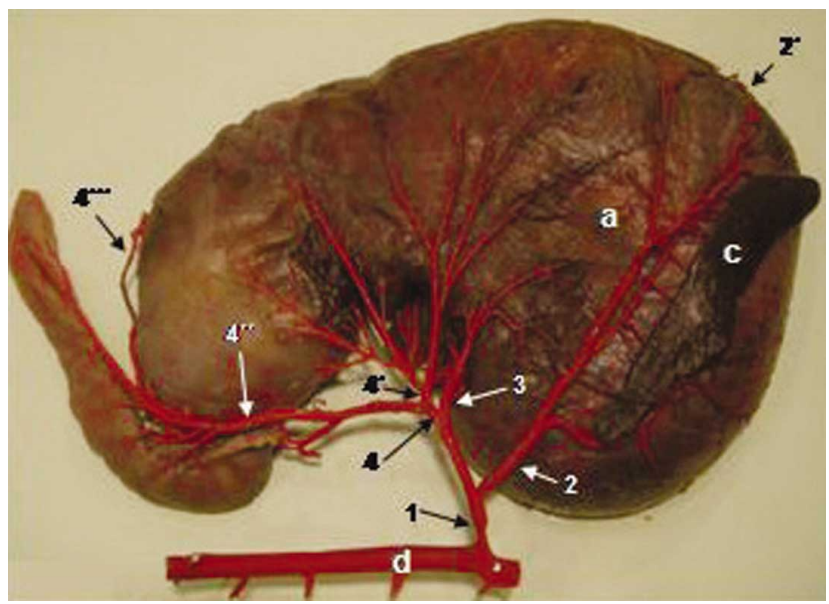

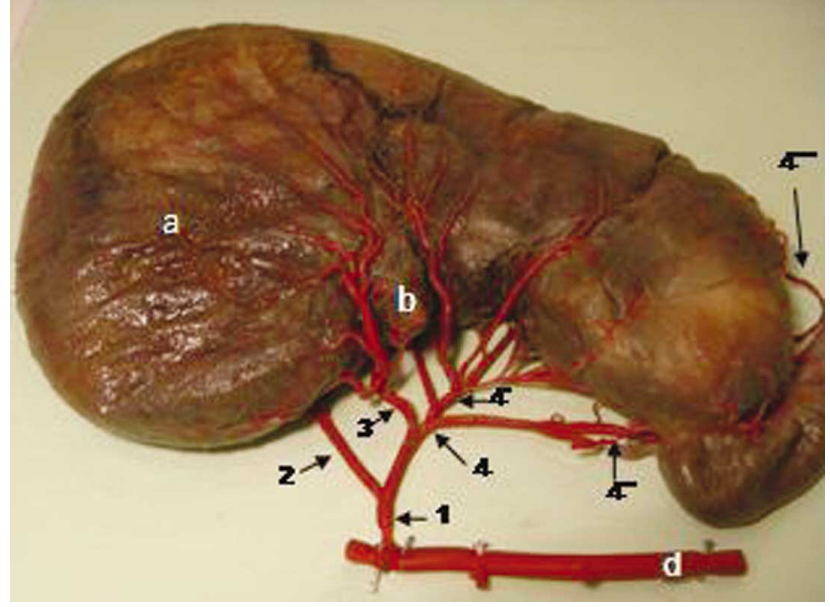

Fig. 8. Cara parietal del estómago del conejo, irrigación arterial proveniente de la arteria celiaca. a. Estómago. b. Esófago. c. Bazo. d. Parte abdominal de la aorta. 1. Arteria celíaca. 2. Arteria esplénica. 2`. Arteria gastroomental izquierda. 3. Arteria gástrica izquierda. 4. Arteria hepática. 4'. Arteria gástrica derecha. 4". Arteria gastroduodenal. 4"”. Arteria gastroomental derecha.

En general estas ramas siguen un patrón común, aunque existen algunas diferencias particulares.

Arteria Esplénica. En conejos la arteria esplénica es la primera en abandonar la arteria celiaca, no así en el perro ya que en ese caso corresponde a la arteria hepática. Cuando llega a las cercanías del fondo del estómago se une al bazo.

En este punto se divide en dos ramas (dorsal y ventral), las que entran por el hilio esplénico por su cara ventral (visceral). La rama dorsal luego de penetrar en el bazo continúa como arteria gastroomental izquierda en la curvatura mayor. La rama esplénica ventral no entra al bazo, dirigiéndose hacia el estómago y genera ramas gástricas cortas. En el perro la rama dorsal origina varias arterias que entran al extremo dorsal del bazo y unas pocas arterias gástricas cortas que se dirigen por el ligamento gastroesplénico hacia la curvatura mayor del estómago en el lado izquierdo. La rama esplénica dorsal continúa como arteria gastroomental izquierda (la que luego se anastomosa con la arteria gastroomental derecha proveniente de la arteria hepática) en la curvatura mayor. La rama esplénica ventral irriga al bazo (Evans \& de Lahunta, 1991).

Al comparar las (Figs. 9 y 10) se observa como la

Fig. 7. Cara visceral del estómago del conejo, irrigación arterial proveniente de la arteria celiaca. a. Estómago. b. Esófago. c. Bazo. d. Parte abdominal de la aorta. 1. Arteria celíaca. 2. Arteria esplénica. 2 = Arteria gastroomental izquierda. 3. Arteria gástrica izquierda. 4. Arteria hepática 4`h Arteria gástrica derecha. 4". Arteria gastroduodenal. 4"'. Arteria gastroomental derecha. 


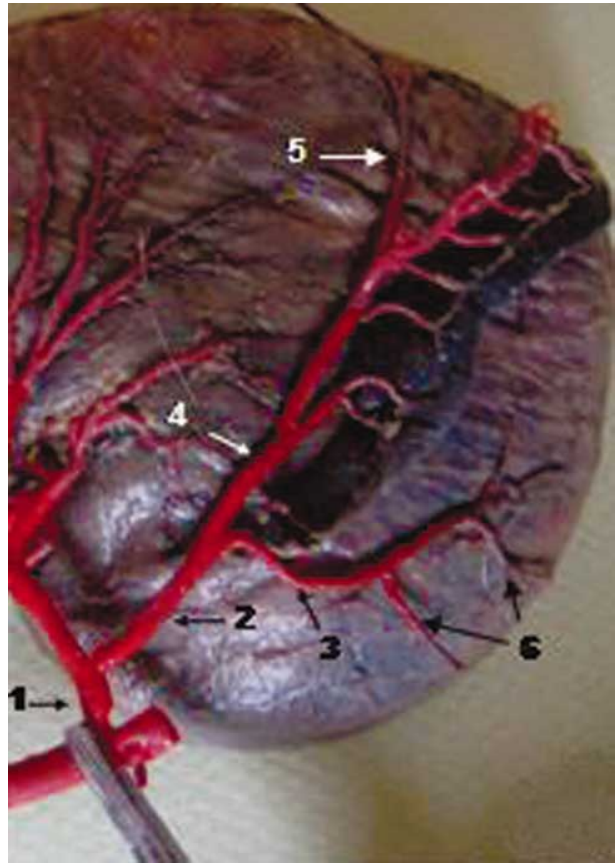

Fig. 9 Ramificación de la artéria esplénica en el conejo. 1. Arteria celíaca. 2. Arteria esplénica. 3. Rama ventral, 4= Rama dorsal, 5. Arteria gastroomental. 6. Arterias gástricas cortas.

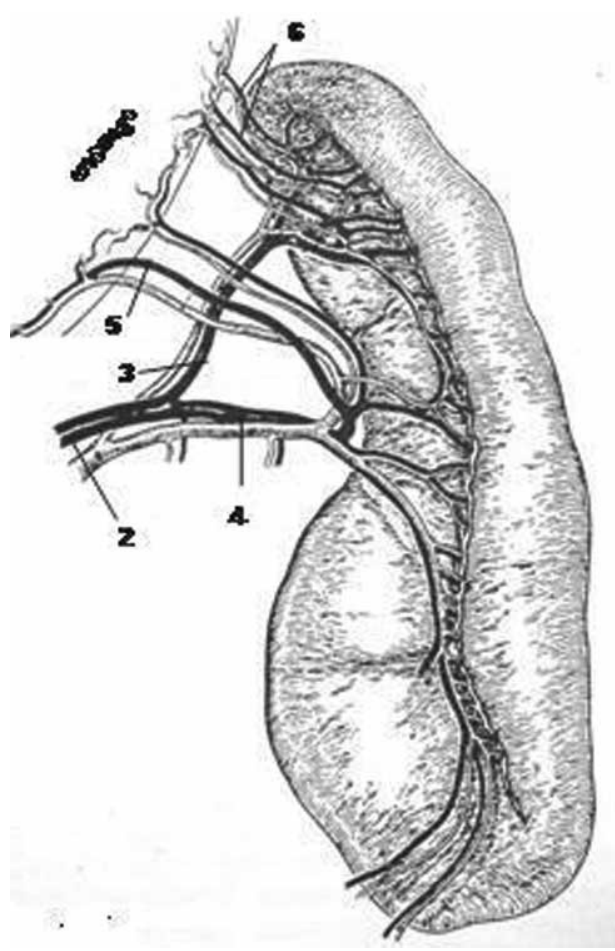

Fig. 10. Esquema de la ramificación de la arteria esplénica en el perro (Nickel et al., 1976). 1. Arteria celíaca. 2. Arteria esplénica. 3. Rama ventral. 4. Rama dorsal. 5. Arteria gastroomental. 6. Arterias gástricas cortas. rama esplénica ventral del conejo no entra al bazo, se dirige hacia el estómago y genera ramas gástricas cortas, por otra parte en el perro no hace este recorrido ya que entra al hilio del bazo y no genera ramas hacia estómago.

Arteria Gástrica Izquierda. En el perro da origen a varias ramas, las que van a irrigar tanto la cara parietal como la cara visceral del estómago. Una o más ramas esofágicas pasan cranealmente hacia el esófago. La arteria gástrica izquierda se extiende, por la curvatura menor, hasta el píloro donde se anastomosa con la arteria gástrica derecha (Evans \& de Lahunta). En el conejo no se observan diferencias significativas, solo no se pudo evidenciar las ramas que irrigan el omento menor, las que en el perro se anastomosan con ramas de la arteria gástrica derecha.

Arteria Hepática. De la arteria hepática del conejo (Fig. 11) emerge una arteria hepática propia que irrigará el hígado, y su división irrigará los distintos lobos del hígado. En el caso del perro a partir de la arteria hepática pueden emerger 3 a 5 arterias denominadas arterias hepáticas propias, las que van a irrigar los distintos lobos del hígado (Evans \& de Lahunta). Las otras ramas de la arteria hepática son similares en el conejo y en el perro, manifiestan la misma distribución y patrón de ramificación.

Arteria gástrica derecha. Se dirige hacia el píloro e irriga la curvatura menor del estómago. La arteria gastroduodenal irriga el píloro, la primera porción del duodeno y de ella nace la arteria gastroomental derecha que va hacia el omento mayor y emite ramas hacia el estómago (arterias gástricas cortas) irrigando la curvatura mayor.

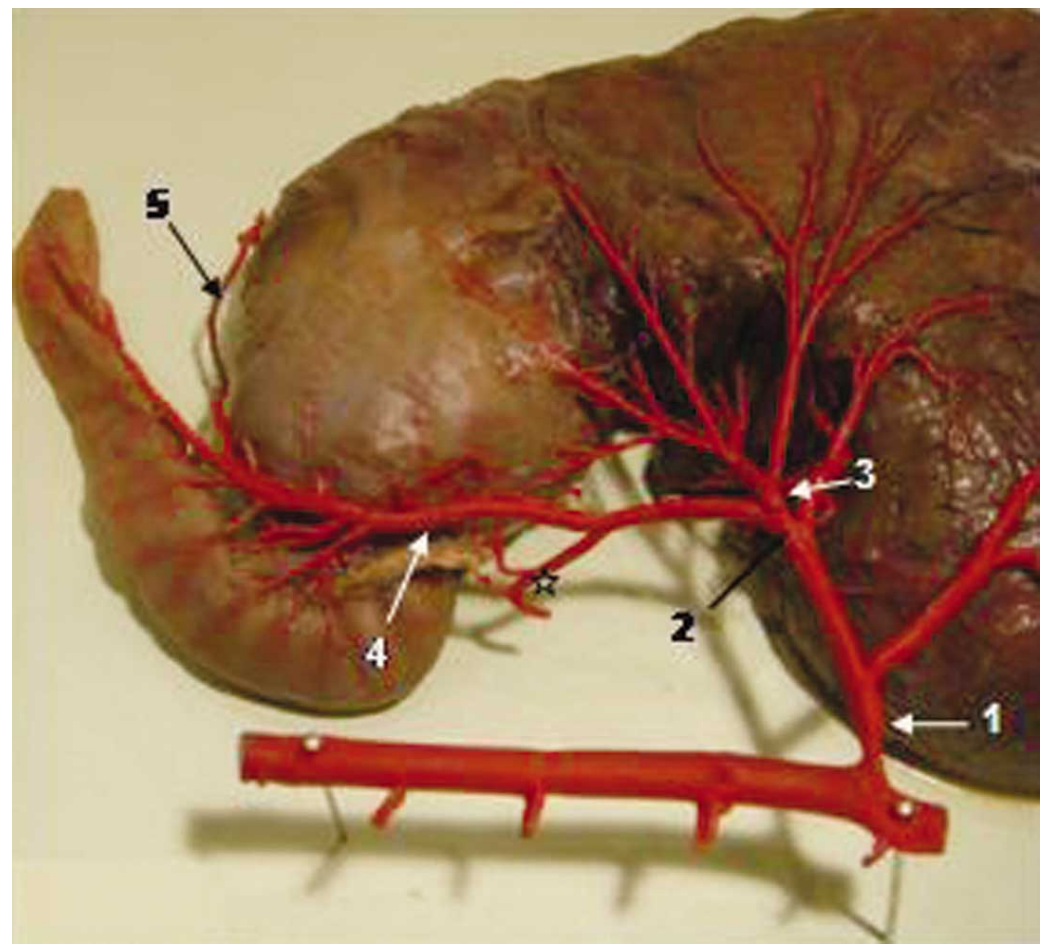

Fig. 11. Ramificación de la arteria hepática del conejo. 1. Arteria celiaca. 2. Arteria hepática. 3. Arteria gástrica derecha. 4. Arteria gastroduodenal. 5. Arteria gastroomental derecha. 6 . Arteria hepática propia. 
De acuerdo a lo observado, al comparar el estómago de conejo con el de perro, se puede concluir que su morfología, ubicación y orientación son similares. Con respecto a la irrigación arterial en ambos órganos, se observó que en ambas especies el patrón de división de los principales vasos que irrigan estos órganos es semejante, con excepción de algunas pequeñas particularidades que no serían significativas para el ejercicio didáctico.

Las principales diferencias entre estas especies se debieron a que el conejo es una especie de hábitos alimenticios distintos al perro. El conejo tiene un ciego muy desarrolla- do, cosa que no ocurre en el perro, por lo que las relaciones entre los órganos en estudio con las otras estructuras que se encuentran dentro del abdomen no son iguales. Estas diferencias no significarían un impedimento en la realización de ensayos quirúrgicos homologables en estomago de estas especies.

En conclusión el estómago del conejo no presenta diferencias anatómicas importantes que impidan la utilización del conejo como modelo experimental para realizar prácticas quirúrgicas básicas, como gastrotomía y suturas viscerales.

HALABI, M. T.; BAHAMONDES, F.; CATTANEO, G.; ADARO, L. \& FLORES, E. Rabbit stomach: animal model for experimental surgery. Int. J. Morphol., 30(1):82-87, 2012.

SUMMARY: Experimental veterinary surgery on small animals -particularly dogs- has restrictions, mainly regarding affect; therefore it is necessary to have a model of the animal to perform such procedures. This model must have anatomic characteristics which homologate those of dogs, but it must also fulfill ease of use and handling. Being a species traditionally used in experimentation, rabbits could be an excellent animal model for this purpose. The goal of this study is to describe the anatomotopography of the stomach of the rabbit and then compare it with the dogs. Three elements were considered: 14 rabbits exposed to various conservation techniques for their later anatomical study, the instruments and supplies to develop such morphological study and a bibliographic record of anatomical parameters of the dog's stomach. The results of this essay show that rabbits have a stomach with a morphology, location and orientation similar to the one of a dog, varying on its relation to other anatomical structures, the irrigation of this organ is as well very similar to the one of the dog. According to what was observed on the stomach, rabbits may be used as an animal model to perform different experimental surgical essays.

KEY WORDS: Rabbit; Stomach; Morphology; Animal model.

\section{REFERENCIAS BIBLIOGRÁFICAS}

Amorim, M. J. A. A. L.; Amorim, J. A. A.; Villarouco, F. M. O.; Ojea, D. B. \& Costa, F J. F. D. Capacidad del estómago de conejos sin raza definida (SRD) (Oryctolagus cuniculus). Rev. Chil. Anat., 19:259-62, 2001.

Dyce, K.; Sack, O. \& Wensing, G. Anatomía Veterinaria. 2a ${ }^{\text {a }}$ ed. Ciudad de México, McGraw-Hill Interamericana, 1999. pp.132-47.

Evans, H. \& De Lahunta, A. Disección del perro. $5^{\mathrm{a}}$ ed. Nueva York, McGraw-Hill Interamericana, 1991. pp.183-220.

Gecele, P. Fisiología digestiva del conejo adulto. Monografías de Medicina Veterinaria, 8:5-12, 1986.

Halabí, M. T. Descripción de estómago y de intestino delgado de conejo (Oryctolagus cuniculus) para su comparación con estómago e intestino delgado de perro. Memoria título Médico Veterinario. Santiago, Chile. Facultad de Ciencias Veterinarias y Pecuarias, Universidad de Chile, 2009.

Nickel, R.; Schummer, A. \& Seiferle, E. Lehrbuch der Anatomie der Haustiere. Berlin, Editorial Paul Parey, 1976. pp.166-77.
Ramirez, I. Estudio epidemiológico descriptivo de casos del servicio de cirugía de animales pequeños Enero 1990-Diciembre 2000. Memoria título Médico Veterinario. Santiago, Chile. Facultad de Ciencias Veterinarias y Pecuarias, Universidad de Chile, 2002.

Ruberte, J. Atlas de anatomía del perro y del gato. Barcelona, Multimédica, 1995.

Sisson, S. \& Grossman, J. Anatomía de los animales domésticos. $5^{\mathrm{a}}$ ed. Ciudad de México, Editorial Ciencia y Cultura Latinoamericana, 1982.

Tompsett, D. Anatomical techniques. $2^{\text {nd }}$ ed. Livingstone, Edinburgh and London, Editorial E\&S, 1970.

Dirección para correspondencia:

Prof. Dra. Estefanía Flores P.

Facultad de Ciencias Veterinarias y Pecuarias

Universidad de Chile, Santiago

CHILE

Email: eflores@uchile.cl 